\title{
Isolation and purification of psoralen and isopsoralen and their efficacy and safety in the treatment of osteosarcoma in nude rats
}

\author{
Honghui Lu , Lihai Zhang ${ }^{¥}$, Daohong Liu, Peifu Tang², Feixiang Song
}

Orthopaedic Department, People's Liberation Army General Hospital, Beijing 100000, China

${ }^{¥}$ Authors Contributed Equally to this Work

\begin{abstract}
Background: Modern studies have shown that psoralen has a significant inhibitory effect on tumor growth in a variety of animals and humans.

Objective: To obtain coumarin compounds - psoralen and isopsoralen - from traditional Chinese medicine Psoralea corylifolia L. using chromatographic techniques and isolation and purification methods, and to observe the transplanted tumor growth inhibitory effects and adverse reactions of psoralen and isopsoralen in nude rats with osteosarcoma.

Methods: Dried ripe fruits of Psoralea corylifolia L. were taken as the raw material to prepare crude extract of Psoralea corylifolia L. by ethanol reflux method. Column chromatography was used to isolate the crude extract; compounds were structurally identified based on ${ }^{1} \mathrm{H}-\mathrm{NMR},{ }^{13} \mathrm{C}$-NMR spectra, the two compounds were identified as psoralen andisopsoralen, and their contents were $99.7 \%$ and 99.6, respectively. Nude rat model of osteosarcoma was established; the rats were randomized into: normal saline group, psoralen low- and high-dose groups, isopsoralen low- and high-dose groups, and cisplatin group. Osteosarcoma volume and weight inhibition rates in nude rats in each group were observed; radioimmunoassay was used to determine the serum alkaline phosphatase activity; peripheral blood cell and bone marrow nucleated cell counts were determined; light microscopy was used to observe heart, liver, spleen, lung, kidney, and tumor histopathology; and electron microscopy was used to observe the fine structure of tumor cells.

Results: Tumor volume inhibition rates were $43.75 \%$ and $40.18 \%$, respectively, in the psoralen and isopsoralen low-dose groups, and tumor weight inhibition rates were $38.83 \%$ and $37.77 \%$. Tumor volume inhibition rates were $67.86 \%$ and $66.96 \%$, respectively, in the psoralen and isopsoralen high-dose groups, and tumor weight inhibition rates were $49.47 \%$ and $47.87 \%$. Psoralen and ispsoralen markedly lowered serum AKP level. Psoralen and isopsoralen induced apoptosis or necrosis of osteosarcoma. After administration of high doses of psoralen and isopsoralen, toxic reactions such as writhing, lassitude, and hypoactivity were seen. Kidney histopathology showed tubulointerstitial dilatation and congestion, and inflammatory cell aggregation in the renal intercellular space. Psoralen and isopsoralen did not cause any significant toxic side effects to the bone marrow, or other organs such as heart, lung, liver, and spleen.
\end{abstract}

Conclusion: Psoralen and isopsoralen have growth inhibitory effects on transplanted tumor in nude rats with osteosarcoma, and can induce tumor cell apoptosis or necrosis, without significant toxic effects.

Key words: Psoralea corylifolia L., psoralen, isopsoralen, osteosarcoma

DOI: http://dx.doi.org/10.4314/ahs.v14i3.20

\section{Introduction}

Chinese medicine $\mathrm{Bu} \mathrm{Gu} \mathrm{Zhi}$, or $\mathrm{Hu} \mathrm{Jiu} \mathrm{Zi}$, is the dried ripe fruit of Psoralea corylifolia L. in the family Fabaceae. It is warm in nature, pungent in taste, and has the

\section{Corresponding author: \\ Peifu Tang \\ Orthopaedic Department, \\ People's Liberation Army \\ General Hospital, Beijing 100000, China \\ EMail: poivcse5@163.com}

actions of reinforcing kidney to strengthen yang, warming middle energizer to stop diarrhea, and relieving dyspnea. Psoralen and isopsoralen are its major active constituents, ${ }^{1-2}$ which are effective drugs for tumor-like dermatoses. Modern studies have shown that psoralen has a significant inhibitory effect on tumor growth in a variety of animals and humans. ${ }^{3-5}$ Therefore, the study of isolation and purification of psoralen and ispsoralen and their effects in tumor treatment is of important significance.

\section{Materials}

Reagents and drugs

Psoralea corylifolia L. (dried seeds), were purchased from 
Dalian Haishengyuan Pharmacy, harvested in Henan, fried products, identified by Professor Wang Mang of the Institute of Botany stored in Beijing herbarium center, CAS as the dried ripe fruit of Psoralea corylifolia L. in the family Fabaceae. Psoralen (2012-0623-5) and isopsoralen (2013-0215-6) reference substances, were purchased from Shanghai Meilun Biotechnology Co., Ltd., purity of $98.98 \%$; solvents such as ethanol, n-hexane, ethyl acetate and methanol used in the experiment were of analytical grade.

\section{Instruments}

The instruments used included: Agilent model 2100 HPLC System, Agilent Technologies, USA; model 2563B UV detector, model 1050 thermostatic circulator, Shanghai Bokang Experimental Instrument Co., Ltd.

\section{Animals}

We used 4 6-week-old female nude rats, weighing 18 20 g, purchased from the SPF Laboratory Animal Center of Chinese Medical University, animal certificate No.: YKD (CN) 2013-0509.

\section{Cell lines}

SD rat osteosarcoma UMR-106 cell lines were from the Peking Union Medical College Hospital, China.

\section{Methods}

\section{Extraction of psoralen and isopsoralen}

Psoralea corylifolia L. seeds were pulverized, and passed through a 60 mesh sieve, $10 \mathrm{~g}$ of the powder was taken, and soaked 3 times at room temperature in 50\% ethanol $(300 \mathrm{ml}, 150 \mathrm{ml}, 150 \mathrm{ml}$ ), with each time lasting 2 hours. The collected leaching solution was reduced to $1 / 2$ volume, kept overnight, and filtered after completion of precipitation. The precipitate was thoroughly dissolved with a 10-fold amount of methanol, decolorized by addition of active carbon, and filtered; after removal of methanol, the remaining was kept overnight, and crystallized to give $2 \mathrm{~g}$ of white needle crystals (i.e. Psoralea corylifolia L. coumarins).

\section{Preparation of expanded test for psoralen and isopsoralen}

(Soaking, percolation: $20 \mathrm{~kg}$ of crude Psoralea corylifolia L. powder was placed in a ceramic bowl, infiltrated with $50 \%$ ethanol, then loaded into a percolation pot, added with $50 \%$ ethanol until the drug surface was exceeded, then soaked overnight. The next day, the drug was percolated; ethanol amount was equivalent to 8 times the amount of raw materials, which was about $160 \mathrm{~L}$.
Removal, concentration: The percolate was concentrated to $1 / 3$ of the original volume through membrane, and ethanol was removed.

Placement, filtration: The concentrated solution was left overnight for precipitation, then filtered with filter cloth, the precipitate was brown and resinous.

Methanol extraction: The precipitate was extracted under reflux several times with a 25-fold amount of methanol, the extracts were then combined, and decolorized by addition of active carbon $2 \%$ the weight of the precipitate. Methanol was removed, and the remaining was concentrated, left for crystallization, then filtered to give crude product, the mother solution was concentrated again to yield some crude product, followed by drying at $80^{\circ} \mathrm{C}$. A total of $154 \mathrm{~g}$ of crudeproduct was obtained.

Recrystallization: Crude product was fully dissolved by heating under reflux with a crude product/methanol proportion of $3: 100$, then decolorized by addition of active carbon $1 \%$ the amount of crude product (reflux for $30 \mathrm{~min}$ ), filtered, left for crystallization, then filtered again, the mother solution was reduced to $1 / 3$, and left for crystallization, after successive processing, several batches of crystals were obtained, which were dried at $80^{\circ} \mathrm{C}$ to give $131 \mathrm{~g}$ of fine product.

\section{Isolation of psoralen and isopsoralen}

120 gof $120 \sim 200$ mesh chromatographic neutral alumina was taken, $2 \mathrm{~g}$ of Psoralea corylifolia L. coumarin crystals were dissolved in a small amount of hot methanol, mixed with $6 \mathrm{~g}$ of alumina, and dried in the air at room temperature. After dry column chromatography with 15 drops of acetone per $50 \mathrm{ml}$ of benzene/petroleum ether (40:10) as the eluent, the sample was observed under UV light, two fluorescence bands were separately selected for extraction under reflux with methanol, after solvent was reduced to a small volume, the remaining was crystallized, and filtered to give crystals I and II.

\section{Identification of psoralen and isopsoralen}

Compound I: colorless needle crystals, mp 163 $\sim 164{ }^{\circ} \mathrm{C}$. TOF-MS m / z: $187[\mathrm{M}+\mathrm{H}]^{+}$; 1H-NMR $(400 \mathrm{MHz}, \mathrm{CD} 3 \mathrm{COCD} 3) \delta: 6.37(1 \mathrm{H}, \mathrm{d}, \mathrm{J}=9.6 \mathrm{~Hz}$, 3-H), $6.99\left(1 \mathrm{H}, \mathrm{d}, \mathrm{J}=1.2 \mathrm{~Hz}, 3^{\prime}-\mathrm{H}\right), 7.57(1 \mathrm{H}, \mathrm{s}, 8-\mathrm{H})$, $8.05\left(1 \mathrm{H}, \mathrm{d}, \mathrm{J}=2.2 \mathrm{~Hz}, 2^{\prime}-\mathrm{H}\right), 8.10(1 \mathrm{H}, \mathrm{d}, \mathrm{J}=9.6 \mathrm{~Hz}$, 4-H); 13C-NMR (100 MHz, CD3COCD3) $\delta: 160.9$ (C-2), 157.7 (C-7), 152.9 (C-9), 147.7 (C-2'), 146.0 (C-4), 125.7 (C-6), 121.5 (C-5), 117.4 (C-10), 115.2 (C-3), 107.4 
(C-3'), 99.8 (C-8). The above data were consistent with the psoralen reported in the literature. ${ }^{6}$

Compound II: white needle crystals, mp 102 $103{ }^{\circ} \mathrm{C}$. TOF-MS m / z: $187[\mathrm{M}+\mathrm{H}]^{+} ;{ }^{1} \mathrm{H}-\mathrm{NMR}(400$ $\left.\mathrm{MHz}, \mathrm{CD}_{3} \mathrm{COCD}_{3}\right) \delta: 6.38(1 \mathrm{H}, \mathrm{d}, \mathrm{J}=9.6 \mathrm{~Hz}, 3-\mathrm{H})$, $7.79(1 \mathrm{H}, \mathrm{d}, \mathrm{J}=9.6 \mathrm{~Hz}, 4-\mathrm{H}), 7.38(1 \mathrm{H}, \mathrm{d}, \mathrm{J}=8.4 \mathrm{~Hz}$, 6-H), $7.68\left(1 \mathrm{H}, \mathrm{d}, \mathrm{J}=2.1 \mathrm{~Hz}, 2^{\prime}-\mathrm{H}\right), 7.09$ (1H, d, J = 2.1 $\left.\mathrm{Hz}, 3{ }^{\prime}-\mathrm{H}\right) ;{ }^{13} \mathrm{C}-\mathrm{NMR}\left(100 \mathrm{MHz}, \mathrm{CD}_{3} \mathrm{COCD}_{3}\right) \quad \delta: 160.7$ (C-2), 157.3 (C-7), 148.0 (C-9), 145.2 (C-2'), 145.2 (C-4), 109.7 (C-6), 121.3 (C-5), 114.4 (C-10), 115.2 (C-3), 104.4 (C-3'), 117.9 (C-8). The above data were consistent with the isopsoralen reported in the literature. ${ }^{6}$

Fig. 1: Structural formulas of psoralen and isopsoralen compounds

HPLC purity analysis of psoralen and isopsoralen Chromatographic column: Agilent ZORBAX SB-C18 $(250 \times 416 \mathrm{~mm}$, i.d. $5 \mu \mathrm{m})$; mobile phase: methanol/ water $=50: 50$, flow rate: $1.0 \mathrm{~mL} / \mathrm{min}$, sample injection volume: $10 \mu \mathrm{L}$; column temperature: $30^{\circ} \mathrm{C}$; detection: using diode array detector (DAD); detection wavelength: $280 \mathrm{~nm}$. Identification was performed using psoralen and isopsoralen standard substances. The purity of resulting psoralen was $99.7 \%$, and isopsoralen $99.6 \%$.

\section{Anti-osteosarcoma experiments of psoralen and isopsoralen}

Establishment of nude rat model of osteosarcoma UMR-106 cell tibial transplanted tumor

The model was established referring to Fisher's method: ${ }^{7}$ nude rats were anesthetized, disinfected, UMR-106 cell suspension was injected into the marrow cavity of cortical bone in the tuberosity region in the lower part of tibia, injection volume was approximately $0.2 \mathrm{~mL}$ (containing $2 \times 10^{6}$ cells).

\section{Animal grouping, administration and general} observation

Fifty rats were successfully modeled, which were divided into normal saline group; psoralen low dose (PLD) group and isopsoralen low dose (IPLD) group $320 \mu \mathrm{g} /(\mathrm{kg} \cdot \mathrm{d})$; psoralen high dose (PHD) group and isopsoralen high dose (IPHD) group $1600 \mu \mathrm{g} /(\mathrm{kg} \cdot \mathrm{d}) ;$ and cisplatin (DDP) group $2 \mathrm{mg} / \mathrm{kg}$. Intratumoral multi-point injection began when the tumor grew to 0.5 $\mathrm{cm} \times 0.5 \mathrm{~cm}$. All groups except for DDP group were administered QD for 2 courses, where 5 days constituted 1 course, administration was discontinued for 1 day between the two courses. DDP group was administered a total of four times on the 1st, 4th, 7th, and 10th days. Mental conditions, activity, body weight (BW), eating, drinking, urination, and defecation in nude rats in each group were observed. On the next day after withdrawal of administration, rats were sacrificed for collection of tissue samples. The tumor, heart, liver, spleen, lung, and kidney tissues of nude rats were $\mathrm{HE}$ stained and cut into sections for pathological examination, the tumor tissue sections were examined using transmission electron microscopy.

Tibial transplanted tumor growth inhibitory effects of psoralen and isopsoralen in nude rats with osteosarcoma

(1) Calculation of tumor volume inhibition rate (VIR): rats were sacrificed, tumors were removed, and longest diameter (a) and shortest diameter (b) of tumors were measured. Tumor volume $(T V)=a \times b^{2} / 2$; tumor VIR $=($ TV of control group - TV of experimental group) / TV of control group $\times 100 \%$;

(2) Calculation of tumor weight inhibition rate (WIR): the tumors removed, and tumor weight (TW) was measured, tumor WIR $=$ (TW of control group TW of experimental group) / TW of control group $\times$ $100 \%$;

(3) Evaluation of tumor inhibitory effect: referring to the literature, ${ }^{9}$ the anti-tumor effects of drugs were evaluated with tumor VIR and WIR as indicators, efficacy evaluation criteria: ineffective if IR $<30 \%$; effective if IR $\geq 30 \%$, and $\mathrm{P}<0.05$.

Determination of the effects of psoralen and isopsoralen on serum alkaline phosphatase (AKP) activity, peripheral blood cells and bone marrow nucleated cells in nude rats

Blood was sampled from the orbital venous plexus of nude rats in each group, sera were collected, and AKP activity in serum was determined by radioimmunoassay.

$0.5 \mathrm{~mL}$ of orbital venous blood was taken from nude rats, placed into anticoagulant test tubes, and peripheral blood cells of nude rats were determined using automatic hematology analyzer within $6 \mathrm{~h}$.

Nude rats were sacrificed, and right femurs were removed, bone marrow were washed in PBS, and blown and mixed uniformly, $10 \mu \mathrm{L}$ of which was added into $190 \mu \mathrm{L}$ of $2 \%$ glacial acetic acid solution, then $10 \mu \mathrm{L}$ was taken for counting under a microscope with cell counting chamber, formula: number of bone marrow nucleated cells $($ cells $/ \mathrm{L})=[$ (sum of number of nucleated cells in four grids / 4) $\left.\times 10 \times 20 \times 10^{8}\right] / \mathrm{L}$. 


\section{Statistical analysis}

Determination results were statistically analyzed using SSPS 15.0 software, all measurement data were expressed as mean \pm standard deviation $(\mathrm{x} \pm \mathrm{s})$. Comparison among multiple groups was performed firstly by homogeneity test of variance, then, if the variances were homogeneous, one-way ANOVA was used; and if the variances were not homogeneous, grouped rank sum test (Kruskal-Wallis method) was used.

\section{Results}

Observation of general conditions of experimental nude rats

During the experiment, nude rats in each experimental group were in good condition, and acted normally, without significant reduction in body weight; after administration of drugs, writhing movements were seen within a few minutes in nude rats in the PHD and IPHD groups, from the 3rd day of administration, lassitude, hypoactivity, and other toxic reactions appeared, but there was no significant reduction in body weight; from the 3rd day of administration, the nude rats in the DDP group presented significant body weight loss, listlessness, lack of activity, and reduced food and water intake, without showing writhing movements. No other abnormalities were found in nude rats in other groups.

Effects of psoralen and isopsoralen on body weight and tibial osteosarcoma growth in nude rats

Each dose group of psoralen and isopsoralen had little influence on the body weight of osteosarcomabearing nude rats; body weight of nude rats in the DDP group significantly decreased. Osteosarcoma volume and weight markedly reduced in nude rats in the PLD and IPLD groups. The inhibitory effects were more pronounced in nude rats in the PHD, IPHD and DDP groups (see Tab. 1).

Tab. 1 Effects of psoralen and isopsoralen on body weight and tumor growth in osteosarcoma-bearing nude rats $(\mathrm{n}=8, \mathrm{x} \pm \mathrm{s})$

\begin{tabular}{cccccc}
\hline Group & BW $(\mathrm{g})$ & TV $\left(\mathrm{cm}^{3}\right)$ & VIR $(\%)$ & TW $(\mathrm{g})$ & WIR $(\%)$ \\
\hline NS & $20.45 \pm 1.20$ & $1.12 \pm 0.70$ & 0 & $1.88 \pm 0.90$ & 0 \\
DDP & $17.00 \pm 1.39 *$ & $0.36 \pm 0.25^{*}$ & 67.86 & $0.75 \pm 0.39 *$ & 60.11 \\
PLD & $19.80 \pm 1.00 \#$ & $0.63 \pm 0.33^{* *}$ & 43.75 & $1.15 \pm 0.36^{* *}$ & 38.83 \\
PHD & $19.39 \pm 1.20 \#$ & $0.36 \pm 0.21^{*}$ & 67.86 & $0.95 \pm 0.52^{* *}$ & 49.47 \\
IPLD & $19.76 \pm 0.93 \#$ & $0.67 \pm 0.23^{* *}$ & 40.18 & $1.17 \pm 0.32^{* *}$ & 37.77 \\
IPHD & $19.40 \pm 1.18 \#$ & $0.37 \pm 0.13^{*}$ & 66.96 & $0.98 \pm 0.45^{* *}$ & 47.87 \\
\hline
\end{tabular}

Note: $* \mathrm{P}<0.01$ compared with NS group; ${ }^{* *} \mathrm{P}<0.05$ compared with NS group; \# $\mathrm{P}>0.05$ compared with NS group

Effects of psoralen and isopsoralen on serum AKP activity in nude rats bearing tibial transplanted osteosarcoma
The results showed that psoralen and isopsoralen could markedly decrease serum AKP level in osteosarcomabearing nude rats (see Tab. 2). 
Tab. 2 Effects of psoralen and isopsoralen on serum AKP in osteosarcoma-bearing nude rats $(\mathrm{x} \pm \mathrm{s})$

\begin{tabular}{lll}
\hline Group & Dose & AKP $(\mathrm{U} / \mathrm{L})$ \\
\hline NS & $2 \mathrm{~mL}$ & $1567.45 \pm 870.68$ \\
DDP & $2 \mathrm{mg} / \mathrm{kg}$ & $512.20 \pm 143.11^{*}$ \\
PLD & $320 \mu / \mathrm{kg}$ & $812.34 \pm 205.56^{* *}$ \\
PHD & $1600 \mu / \mathrm{kg}$ & $514.89 \pm 189.23^{*}$ \\
IPLD & $320 \mu / \mathrm{kg}$ & $806.36 \pm 196.36^{* *}$ \\
IPHD & $1600 \mu / \mathrm{kg}$ & $513.69 \pm 196.21^{*}$ \\
\hline
\end{tabular}

Note: $* \mathrm{P}<0.01$ compared with NS group; $* * \mathrm{P}<0.05$ compared with NS group

Histopathological examination of tumor, heart, liver, spleen, lung, and kidney tissues of osteosarcoma-bearing nude rats

Tumors were generally large in the NS group, with the sections in a "fish-like" shape. Rich capillaries were observed under the light microscope, cells were distributed densely and disorderly, nuclei differed in sizes, with some in abnormal shapes, the number of nucleoli ranged between $1 \sim 4$, and mitosis was common. Intratumoral ossification was evident, and relatively many cartilaginous tissues could be seen.

In the experimental groups and DDP group, tumor cell density decreased, large amount of necrotic tumor cells appeared, protein-like substances and cell debris could be observed in the center, cells shrank, nuclei were condensed, and mitosis was reduced. Hemorrhage was observed in some tumor tissues, intercellular substances increased significantly, and lymphocyte infiltration was seen. Necrotic tumor tissues gradually increased with increasing doses in the experimental groups.

No metastatic lesion was found in the abdomen, liver, and lung of animals in the treatment groups. There were no significant changes in the color and texture of organs such as liver, heart, spleen, lung and kidney. Histopathological examination showed tubulointerstitial vascular dilatation and congestion, and aggregation of inflammatory cells in the renal intercellular space in the PHD, IPHD and DDP groups.

Electron microscopic observation of the effects of psoralen and isopsoralen on osteosarcoma tissue structure in nude rats

In the NS group, chromatin of tumor cells were evenly distributed, nuclei were enlarged and relatively light, mitochondria were relatively small, and were in irregular polygonal shapes, lipid droplets were contained in the cytoplasm. In the PLD and IPLD groups, cells were arranged relatively loosely, and relatively large amount of endoplasmic reticulum were dilated, typical necrotic features could be seen, nuclear membranes were damaged and dissolved, nuclei were condensed, a large amount of vacuoles were seen in the cytoplasm, some of the cytoplasm were dissolved, organelles were rarely observed, and chromatins were distributed in clumps. In the PHD and IPHD groups, cells were ruptured, cytoplasm were loose and disappeared, nuclear membranes were ruptured and rough, intranuclear structure was blurred, chromatins were loose, and mitochondria were relatively large. In the DDP group, mitochondria and matrices were cavitated and denatured, nuclei were homogenized, color was deepened, chromosomes were distributed in clumps, and lipid droplets were in flocculent shape.

\section{Determination of blood cell and bone marrow nucleated cell counts in nude rats in each group}

The numbers of peripheral red blood cells, hemoglobin, white blood cells, and platelets of nude rats in the psoralen and isopsoralen groups were not significantly different compared with the NS group $(\mathrm{P}>0.05)$. White blood cells markedly decreased in the DDP group $\left(2.13 \pm 0.36 \times 10^{9} / \mathrm{L}\right)$, whichwas statistically significant compared with the NS group $\left(3.89 \pm 1.73 \times 10^{9} / \mathrm{L}\right)(\mathrm{P}$ $<0.01)$, the numbers of red blood cells, hemoglobin, and platelets were not significantly different compared with the NS group (P > 0.05).

The number of bone marrow nucleated cells markedly decreased in the DDP group $(41.83 \pm 6.90) \times$ $10^{10} /$ Lr compared with the NS group $(94.08 \pm 10.82)$ $\times 10^{10} / \operatorname{Lr}(\mathrm{P}<0.01)$. The numbers of bone marrow nucleated cells in other groups were not statistically significant compared with the NS group $(\mathrm{P}>0.05)$. 


\section{Discussion}

During the experiment, after inoculation of UMR106 cells, visible lumps appeared in the tibial part of left hind limbs in all nude rats in about 4 days, the tumor diameter increased to around $0.5 \mathrm{~cm}$ in about 7 days, inoculation success rate was 100\%. During the experiment, tumor growth was relatively rapid in nude rats in the NS group, with some tumors showing ulceration tendencies. Psoralen and isopsoralen groups had significant inhibitory effects on osteosarcoma in nude rats, without the presence of obvious toxic side reactions. The osteosarcoma inhibitory effect became increasingly apparent with increasing doses in each experimental group.

AKP protein is an important protein produced by osteoblasts, in osteosarcoma, not only the osteoblasts are active in terms of number and function, serum AKP activity is also elevated. Studies have shown that, ${ }^{10-12}$ osteosarcoma patients with increased AKP have relatively poor prognosis in terms of metastasis and survival rates. Bramer et al.'s study has shown that after receiving preoperative neoadjuvant chemotherapy, serum AKP level of osteosarcoma patients was also significantly correlated with the survival time. ${ }^{13}$ Patients with decreased AKP were relatively well susceptible to drugs, and had a better prognosis. Serum AKP gradually decreased with increasing doses in each treatment group, its level was directly proportional to the tumor weight and volume of nude rats, which indicated that psoralen and isopsoralen were especially suitable for osteosarcoma patients with elevated AKP in clinical settings, the preoperative use of psoralen or isopsoralen can reduce blood AKP level, so that the tumor is confined for easy removal, thereby reducing the postoperative recurrence and metastasis rates.

Clinical evaluation of the efficacy of nonsurgical therapies for tumors often investigates whether they can cause tumor apoptosis or necrosis, as well as the speed and range of tumor necrosis as key indicators. Light and electron microscopy showed pronounced apoptosis or necrosis of osteosarcoma lumps in nude rats in the PHD and IPHD groups, and the apoptotic and necrotic areas increased with increasing doses of melittin. It is thus clear that psoralen and isopsoralen have important significance in cancer treatment.

With respect to toxic side reactions, the numbers of bone marrow nucleated cells and peripheral white blood cells in the treatment groups did not statistically differ from the NS group, but were markedly reduced in the DDP group, indicating that DDP had bone marrow suppression side effects, while psoralen and isopsoralen did not. Histopathological examination results showed no obvious hemorrhage, necrosis or tumor metastasis in vital organs (the heart, liver, spleen, and lung) of nude rats in each experimental group. Nevertheless, renal tubular cytoplasmic edema and denaturation, accumulation of inflammatory cells in renal intercellular space were seen in kidneys of nude rats in the PHD and IPHD groups, resulting in side effects. The results of the present study demonstrates that psoralen and isopsoralen have significant inhibitory effects on osteosarcoma in nude rats, without the presence of obvious toxic side effects within therapeutic doses. Clinically, psoralen or isopsoralen can be applied in combination with other methods to improve their antitumor effects and reduce their toxic side effects, and to achieve the goal of "eliminating pathogens while not damaging normal cells", thereby laying the foundation for the inclusion of psoralen and isopsoralen into the clinical treatment of osteosarcoma.

\section{References}

1. Zhu ZR, Li Y, Liu J, Xiao R. The study of Psoralen antioxidant effects of flavonoids. Chinese Journal of Veterinary Drug. 2005; 39(2): 18-20.

2. Herrmann G, Brenneisen P, Wlaschek M, Wenk J, Faisst K, Quel G, Hommel C, Goerz G, Ruzicka T, Krieg T, Sies H, ScharffetterKochanek K. Psoralen photoactivation promotes morphological and functional changes in fibroblasts in vitro reminiscent of cellularsenescence. Journal of Cell Science. 1998; 111(6): 759-767.Xu XK, Hu J, Liu RH. Study of the chemical constituents of radix of Ficus caric L.. Pharmacentical Care and Research. 2005; 5(2): 138-138.

7. Fisher JL, Mackie PS, Howard ML, Zhou H, Choong PF. The expression of the uroki-nase plasminogen activator system in metastatic murine osteosarcoma. Clin Cancer Res. 2001; 7(6): 1654-1660.

8. Ling CQ, Huang XQ, Liu L. Study on less toxic and better tumoricidal effects of sustainedrelease preparation of melittin by local intratumor injection. Academic Journal of Second Military Medical University. 2001; 22(7): 615-616.

9. Kurdow R, Boehle AS, Haye S. Ganciclovir prodrug therapy is effectsive in a murine xenotransplant model of human lung cancer. Ann Thorac Surg. 2002; 73(3): 905-910.

10. Bacci G, Longhi A, Ferrari F, Briccoli A, Versari M, Picci P. Adjuvant and neoadjuvant chemotherapy for osteosarcoma of the extremities: 27 year experience at Rizzoli Institute, Italy. Eur J Cancer. 
2005; 41(18): 2836-2845.

11. Bacci G, Longhi A, Ferrari S, Lari S, Manfrini M, Donati D, Forni C, Versari M. Prognostic significance of serum alkaline phosphatase in osteosarcoma of the extremity treated with neoadjuvant chemotherapy: recent experience at Rizzoli Institute. Oncol Rep. 2005; 9(1): 171-175.

12. Tang LN, Lin F, Zheng SE. Correlation between serum AKP and prognosis of osteosarcoma (with 69 osteosarcoma cases). China Oncology. 2007; 17(12): 964-967.

13. Bramer JA, Abudu AA, Tillman RM, Carter SR, SumathiVP, GrimerRJ. Pre-and post-chemotherapy alkaline phosphatase levels as prognostic indicators in adults with localised osteosarcoma. Eur J Cancer. 2005; 41(18): 2846-2852. 